\title{
Paternal use of antidepressants and offspring outcomes in Sweden: nationwide prospective cohort study
}

\author{
Alexander Viktorin, ${ }^{1}$ Stephen Z Levine, ${ }^{2}$ Margret Altemus, ${ }^{3}$ Abraham Reichenberg, ${ }^{4,5}$ \\ Sven Sandin ${ }^{1,4,5}$
}

${ }^{1}$ Department of Medical

Epidemiology and Biostatistics, Karolinska Institutet, Stockholm, Sweden

${ }^{2}$ Department of Community Mental Health, University of Haifa, Haifa, Israel

${ }^{3}$ Department of Psychiatry, Yale School of Medicine, New Haven, CT, USA

${ }^{4}$ Department of Psychiatry, Icahn School of Medicine at Mount Sinai, New York, NY 10029, USA

${ }^{5}$ Seaver Autism Center for Research and Treatment at Mount Sinai, New York, NY, USA Correspondence to:

Sven Sandin

sven.sandin@ki.se

Additional material is published online only. To view please visit the journal online.

Cite this as: $B M J$ 2018;361:k2233 http://dx.doi.org/10.1136/bmj.k2233

Accepted: 18 April 2018

\section{ABSTRACT}

OBJECTIVE

To examine the association between paternal antidepressant use at conception and offspring preterm birth, malformations, autism spectrum disorder, and intellectual disability.

DESIGN

Observational prospective cohort study with regression methods, and negative control comparison.

\section{SETTING}

Sweden nationwide.

PARTICIPANTS

170508 children conceived from 29 July 2005 and born in 2006-07, followed up to 2014 at age 8-9 years. This cohort included 3983 children born to fathers receiving antidepressant treatment during the conception period (that is, from four weeks before conception to four weeks after), a control group of 164492 children not exposed to paternal antidepressant use, and a negative control comparison group of 2033 children born to fathers who did not use antidepressants during the conception period but began antidepressant treatment later during the pregnancy period (that is, from four weeks after conception to childbirth).

MAIN OUTCOME MEASURE

Offspring preterm birth, malformation diagnosed at birth, diagnosis of autism spectrum disorder, and diagnosis of intellectual disability.

RESULTS

Paternal antidepressant use during conception was not associated with preterm birth (adjusted odds ratio 0.91 (95\% confidence interval 0.79 to 1.04)) or malformations (1.06 (0.90 to 1.26)) using

\section{WHAT IS ALREADY KNOWN ON THIS TOPIC}

Depression is currently the leading cause of disability worldwide according to the World Health Organization, with ensuing high rates of antidepressant treatment

Maternal use of antidepressants during pregnancy has been extensively investigated, and although some research suggests antidepressant treatment might adversely affect sperm, paternal treatment at conception has received little attention

\section{WHAT THIS STUDY ADDS}

Paternal intake of antidepressants around the time of conception might not increase the risk of four major adverse outcomes in offspring: preterm birth, malformation, autism, or intellectual disability

This information can assist public health work, help patients and doctors decide on whether to use antidepressants when planning a pregnancy, and help alleviate concerns of fathers who will plan to take antidepressants during conception logistic regression, compared with offspring born to unexposed fathers. No association was seen between antidepressant use during conception and autism (adjusted hazard ratio 1.13 (0.84 to 1.53)) or intellectual disability (0.82 (0.51 to 1.31$))$ using Cox regression. In children whose fathers initiated antidepressant treatment during pregnancy, results were similar for all outcomes apart from intellectual disability, which had an increased adjusted hazard ratio (1.66 (1.06 to 2.59)). Compared with the 2033 children whose fathers initiated antidepressant treatment during pregnancy, the 3983 children exposed to paternal use of antidepressants at conception had no differences in preterm birth, malformation, and autism, but a reduced risk of intellectual disability (adjusted hazard ratio 0.49 (0.26 to 0.93)).

\section{CONCLUSION}

Paternal intake of antidepressants during the period around conception is safe with respect to the risk of the four major adverse outcomes in offspringpreterm birth, malformation, autism, or intellectual disability.

\section{Introduction}

The health of the developing fetus and newborn child is of utmost importance to expecting parents, healthcare providers, and society at large, with preconception and pregnancy health efforts traditionally centred on the mothers. Substantial literature exists on various maternal characteristics during pregnancy (including advanced age, ${ }^{12}$ vitamin supplementation, ${ }^{3}$ and exposure to drug treatment ${ }^{4-13}$ ), maternal pregnancy complications, ${ }^{14} 15$ offspring preterm birth, ${ }^{710}$ malformations, ${ }^{16}$ and neuropsychiatric disorders, ${ }^{1-9}$ among others. Depression is currently the leading cause of disability worldwide according to the World Health Organization, ${ }^{17}$ with ensuing high rates of antidepressant prescriptions. ${ }^{18}$ The effects of maternal antidepressant treatment during pregnancy on short and long term outcomes in offspring has been extensively investigated..$^{4-16}$ By contrast, only limited attention has been given to paternal antidepressant use through potential effects on sperm. ${ }^{19-21}$

The paternal contribution to the embryo is nuclear DNA, which can increase the risk of adverse offspring outcomes from inherited genes, de novo mutations, and epigenetic changes, ${ }^{22}$ directly or indirectly through the placenta, which is derived from fetal cells. In vitro research has shown spermicidal action of varying degrees after antidepressant exposure. ${ }^{23}$ Other previous studies have assessed sperm quality as a result of antidepressant toxicity, and have suggested 
that antidepressant drugs could reduce sperm concentration and motility, cause morphological changes, ${ }^{24}$ and increase DNA fragmentation damage. ${ }^{25-28}$ Sperm DNA fragmentation seems to affect human embryos after implantation development, ${ }^{29}$ and environmental genotoxic exposures have been shown to cause de novo mutations in mice. ${ }^{30}$ In people, increased rates of de novo mutations have been linked to autism. ${ }^{31} 32$ Moreover, advanced paternal age is strongly associated with increased rates of de novo mutations in offspring, and advanced paternal age has in turn been associated with autism and intellectual disability in offspring. ${ }^{233}$

Although the quality of evidence related to the antidepressant effects on sperm is weak and is based on small study samples and on case reports, there is growing but limited interest in the effects of paternal drug exposure before conception on birth outcomes. Researchers have associated paternal exposure to antidepressants before conception with a modest increase in risk of preterm birth, ${ }^{20}$ whereas others have observed a mildly increased risk of autism in a study of paternal antidepressant treatment during conception. ${ }^{21}$ Furthermore, paternal antidepressant treatment at the time of pregnancy has been used as a negative control in studies of maternal treatment. ${ }^{7}$

A better understanding of potential adverse effects of paternal antidepressant treatment is relevant to public health policy, clinical care, family planning, and future research. Therefore, we investigated the effects of paternal antidepressant treatment during conception on preterm birth, birth malformation, autism, and intellectual disability, using data from a Swedish nationwide cohort.

\section{Methods}

Population

Our study cohort consisted of all liveborn children with complete information on gestational age at birth and on the identity of both parents, conceived between 29 July 2005 and 31 December 2007 as identified in the Swedish Medical Birth Register. The register includes $99 \%$ of all births nationwide since 1973, and provides information on gestational age at birth that was used

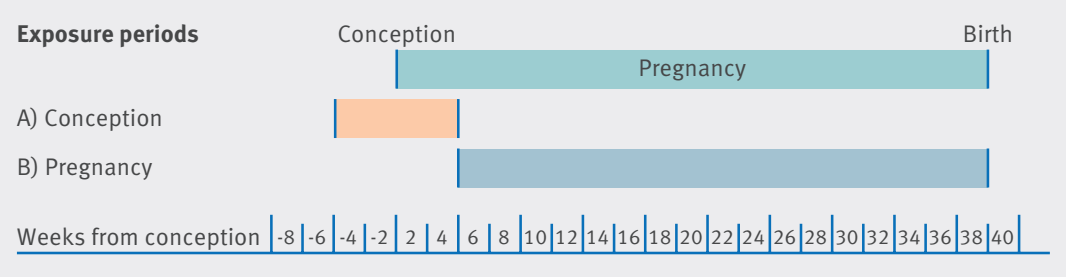

Fig 1 | Description of exposure periods. Three mutually exclusive exposure groups were created in the study: children born to fathers receiving antidepressant treatment during the conception period (A; from four weeks before estimated conception date to four weeks after); children born to fathers receiving no antidepressant treatment during the conception period (A) but who started treatment during the pregnancy period only ( $B$; from four weeks after the estimated conception date to end of pregnancy); and children born to fathers not receiving antidepressant treatment during the conception period or pregnancy period to calculate the date of conception. ${ }^{34}$ In Sweden, 95\% of pregnant women receive early second trimester ultrasonography, which provides the gestational age of the fetus with an error margin of seven days. ${ }^{35}$ Fathers were identified by use of the Multi-Generation Register provided by Statistics Sweden, which separates biological and adoptive parents and excludes parents who do not pass on any information (eg, lack of birth date, or extreme age at birth of the child). ${ }^{3637}$ The study was approved by the regional ethics committee in Stockholm, Sweden (2012/1548/31-1). No individual level consent was required, and all data used were anonymised.

\section{Antidepressant exposure}

TheSwedish Prescribed Drug Registerholdsinformation on all dispensed prescription drugs in Sweden since 1 July 2005, along with the drug name, number of pills per prescription, and an anatomical therapeutic chemical classification system (ATC) code. $^{38} 39$ We identified dispensations of all psychotropic drugs given to both parents (eTables 1 and 2) and calculated treatment periods assuming either one pill was consumed per day for antidepressants or according to the defined daily dose (DDD) provided by WHO for all other psychotropic drugs. Figure 1 describes periods used to classify three mutually exclusive exposure groups:

- Exposure to antidepressants during conception, including offspring born to fathers with at least one dispensation of an antidepressant drug from four weeks before conception to four weeks after (period A in fig 1). Consequently, this group would include offspring born to fathers who started a new antidepressant treatment during this eight week period, and those with fathers with a stable long term treatment regimen overlapping conception, who might have started treatment before the eight week conception period. Moreover, fathers identified as using antidepressants during this period were likely to have continued antidepressant treatment after the conception, overlapping pregnancy and possibly beyond.

- Exposure to antidepressants initiated during pregnancy only, including offspring born to fathers not in group 1-that is, fathers who did not receive antidepressant treatment during the conception period (A in fig 1) but beginning from four weeks after conception to childbirth (B in fig 1). Consequently, this group would only include fathers who started a new antidepressant treatment during pregnancy, because fathers on a stable long term treatment regimen were likely to have overlapped the eight week conception period.

- No exposure to antidepressants, including children of fathers not in the first two groups, who had no antidepressant drug dispensed during conception or pregnancy.

We chose the eight week conception window to account for the final 10 day postmeiotic, late stage 
of spermatogenesis, during which DNA repair cannot occur, ${ }^{40}$ and to account for the margin of error of ultrasound dating. ${ }^{35}$ Effects of potential misclassification due to strict definition of the exposure periods were examined in sensitivity analyses.

\section{Outcomes}

We defined preterm births as having a gestational age of less than 37 weeks. Malformation at birth was identified in the Medical Birth Register (eTable 3), based on a binary variable including any malformation according to ICD-10 (international classification of diseases, 10th revision) codes Q00-99. ${ }^{34}$ Clinically ascertained ICD-10 diagnoses of autism spectrum disorder and intellectual disability were identified from birth to 31 December 2014 in the Swedish National Patient Register, ${ }^{41} 42$ which includes dates of all inpatient psychiatric admissions since 1973 and all outpatient specialist visits since 2001 (eTable 3). ${ }^{41}$

\section{Covariates}

In the current cohort, consistent with previous studies, ${ }^{45943}$ the prevalence of childhood psychiatric disorders increased over time (eFigure 1). Therefore, to adjust for potential confounding due to temporal trends, we included the offspring birth date, coded as number of days from 1 January 2005. Maternal and paternal age are well established risk factors for childhood neuropsychiatric disorders, ${ }^{2}$ and we categorised these ages at childbirth as 20, 20-29, 30-39, and 40 years or above. ${ }^{1}$ Maternal use of antidepressants during pregnancy with psychotropic treatment has previously been associated with many maternal and offspring related outcomes, including pre-eclampsia, ${ }^{15}$ postpartum haemorrhage, ${ }^{14}$ neuropsychiatric disorders, ${ }^{4-13}$ preterm birth, ${ }^{70}$ and malformations ${ }^{16}$; this was included as a binary variable, defined as a dispensation overlapping the pregnancy period.

Genomic studies have identified genetic correlations between many psychiatric disorders, ${ }^{44} 45$ as well as between psychiatric disorders and somatic disorders and offspring birth characteristics. ${ }^{45}$ Therefore, maternal and paternal psychiatric history of 10 separate psychiatric disorder categories (eTable 3) were included, ascertained on the basis of having at least one diagnosis before childbirth in the Swedish National Patient Register. The register includes all Swedish inpatient psychiatric admissions since 1973 and all outpatient specialist admissions since 2001, and provides admission dates along with diagnosis codes in accordance with ICD. ${ }^{41}$ Socioeconomic status was defined by paternal and maternal education levels (duration of education; eTable 4).

\section{Statistical analysis}

We estimated odds ratios of preterm birth and malformations and associated Wald type two sided $95 \%$ confidence intervals by logistic regression. For autism spectrum disorder and intellectual disability, we calculated hazard ratios and associated Wald type two sided 95\% confidence intervals from Cox regression models, which allow for detailed adjustment for censoring affecting the length of follow-up of each child. ${ }^{46}$ Days since birth was used as the underlying time scale. Each child was followed from birth until a diagnosis of the outcome, death, or end of follow-up at 31 December 2014, whichever event occurred first.

All estimates were calculated by models with increasing complexity, beginning with models without adjustment for covariates (model 1), followed by models adjusting for all included potentially confounding covariates (model 2). We calculated the estimates in offspring born to fathers receiving antidepressant treatment during the conception period (A in fig 1) and in offspring born to fathers initiating treatment beginning in the pregnancy period (B in fig 1), compared with offspring of fathers not receiving treatment during conception or pregnancy. Estimates were then calculated in offspring born to fathers receiving antidepressant treatment during the conception period, compared with offspring of fathers initiating treatment in the pregnancy period.

To test the statistical hypothesis of no adverse outcomes in offspring born to fathers receiving antidepressant treatment, the statistical significance was set at the nominal two sided 5\% level. We did not perform any adjustment due to multiplicity of statistical tests. Data management was performed by SAS 9.4, and STATA/IC 14 was used for logistic regression (command logistic) and Cox regression (command stcox).

\section{Sensitivity analyses}

To rule out associations in offspring outcome subgroups, we performed additional analyses in children with mild to moderate intellectual disability, and in those with the most severe type of autism, autistic disorder. The exposure periods were selected prioritising the exposure during conception, which could cause potential misclassification. For example, a father who received antidepressant treatment in the two years before conception but who stopped treatment five weeks before conception would be classified as a non-user, and a father not receiving treatment until three weeks after conception would be classified as being exposed to antidepressants during conception. To examine effects of such potential misclassifications, we analysed more extensive and more restrictive exposure periods.

To rule out risks attributed to specific types of antidepressant drugs, we performed additional analyses for selective serotonin reuptake inhibitor (SSRI) antidepressants of any type, and for each of the four most common SSRI antidepressants. We examined the role of polypharmacy by excluding offspring exposed to non-antidepressant psychotropic drugs. Because data for siblings from the same parents could introduce correlations in the data that are not automatically taken into account when calculating the standard errors and confidence intervals, we performed sensitivity analyses taking into account such correlations through bootstrap techniques. ${ }^{47}$ 
To ascertain the influences of maternal psychiatric disorders and maternal psychotropic medication on our results, we performed sensitivity analyses by excluding offspring born to mothers receiving psychotropic treatment during pregnancy. We investigated potential sex specificity of associations by performing subgroup analyses by offspring sex. The proportional hazards assumption for the Cox regression models was examined by use of Schoenfeld residuals. ${ }^{48}$

Because paternal adherence to treatment was not known, we investigated the proportion of fathers with an antidepressant treatment dispensation overlapping the conception period who did not have a secondary dispensation. Antidepressants are commonly used over longer periods, so one isolated dispensation could indicate that the treatment had not been initiated or was stopped before conception. To examine the effect of single dispensations, we separately analysed fathers with a continuous treatment regimen of antidepressants by including only fathers with at least one additional dispensation within six months. Lastly, fathers exposed to antidepressants only after conception were likely to have incident depression or worse to trigger a prescription (that is, new users), whereas those already receiving antidepressant treatment at conception were more likely to be stable (stable users). The role of this potential difference in new and stable users was examined in analyses confined to users receiving no antidepressant treatment from seven to three months before conception (that is, only newly initiated prescriptions).

\section{Missing values}

Data from 171879 livebirths of children conceived from 29 July 2005 and born up to 31 December 2007 were provided by the Swedish National Board of Health and Welfare. Paternity information was missing for 1314 (0.76\%) children (eTable 5), and gestational age at birth was missing for an additional 57 children; in total, $1371(0.80 \%)$ children did not have complete data and were excluded from the statistical analyses.

\section{Patient involvement}

As mandated by the ethical permission, no attempts were made to contact any cohort members for any aspect of this record linkage study. As such, cohort members were not involved in setting the research question or the outcome measures, nor were they involved in developing plans for the design or implementation of the study. No patients were asked to advise on interpretation or writing up of results. There are no plans to directly disseminate the results of the research to cohort members, but dissemination to the general public and relevant patient groups will be undertaken by using presentations and social media.

\section{Results}

Table 1 presents general descriptive data for the study cohort, and table 2 presents descriptive data for the parents diagnosed as having psychiatric disorders before childbirth. Of 170508 children conceived from 29 July 2005 and born up to 31 December 2007, 3983 (2.3\%) were born to fathers who had dispensed an antidepressant drug within a treatment period overlapping during the conception period, 2033 (1.2\%) were born to fathers without a dispensation overlapping conception but with a dispensation during pregnancy, and 164492 (96.5\%) were born to fathers without a dispensation during conception or pregnancy. Records showed 10689 children (6.3\%) born preterm, 6502 (3.8\%) with birth malformations, $1556(0.9 \%)$ with autism, and $832(0.5 \%)$ with intellectual disability diagnosed between birth and 31 December 2014 when the children were 8-9 years old.

\section{Antidepressant exposure and adverse outcomes}

In the first comparison, 3983 children born to fathers receiving antidepressant drug treatment during the conception period were compared with 164492 unexposed children. In analyses without covariate adjustment (model 1), antidepressant exposure during conception was only associated with an increased risk of autism (hazard ratio 1.39 (95\% confidence interval 1.04 to 1.84)). After covariate adjustment (model 2), no association was observed between antidepressant exposure during conception and any of the outcomes (fig 2).

In the second comparison, 2033 children born to fathers with antidepressant drug treatment beginning during the pregnancy period only were compared with 164492 unexposed children. In analyses without covariate adjustment (model 1), antidepressant exposure after conception and during pregnancy was associated with an increased risk of autism (hazard ratio 1.69 (95\% confidence interval 1.18 to 2.41 )) and intellectual disability (2.14 (1.39 to 3.30); fig 2). After covariate adjustment (model 2), antidepressant exposure after conception and during pregnancy was only associated with an increased risk of intellectual disability (adjusted hazard ratio 1.66 (1.06 to 2.59); fig 2).

In the third comparison, 3983 children born to fathers receiving antidepressant drug treatment during the conception period were compared with 2033 children born to fathers who initiated treatment during the pregnancy period only. In analyses without covariate adjustment (model 1), antidepressant exposure during conception was associated with a decreased risk of intellectual disability (hazard ratio 0.49 (95\% confidence interval 0.27 to 0.91$)$ ), with comparable results remaining after covariate adjustment (model 2; fig 3).

\section{Sensitivity analyses}

Supplemental analyses revealed results that did not deviate quantitatively or qualitatively from the main findings in the conception exposure group in figure 2. These sensitivity analyses looked at:

- Autistic disorder and mild and moderate intellectual disability (eFigure 2)

- Extended conception periods (eFigures 3-6)

- Exposure to SSRI antidepressants specifically (eFigure 7) 


\begin{tabular}{|c|c|c|c|}
\hline \multirow[b]{2}{*}{ Characteristics (No (\%)) } & \multicolumn{3}{|c|}{ Paternal antidepressant treatment } \\
\hline & Unexposed* & Conceptiont & Pregnancy $¥$ \\
\hline No of offspring & $164492(96.5)$ & $3983(2.3)$ & $2033(1.2)$ \\
\hline Offspring with autism spectrum disorder & $1476(0.9)$ & $49(1.2)$ & $31(1.5)$ \\
\hline With autistic disorder & $906(0.6)$ & $25(0.6)$ & $21(1.0)$ \\
\hline Offspring with intellectual disability & $791(0.5)$ & $20(0.5)$ & $21(1.0)$ \\
\hline With mild to moderate intellectual disability & $696(0.4)$ & $16(0.4)$ & $20(1.0)$ \\
\hline Offspring born preterm & $10319(6.3)$ & $251(6.3)$ & $119(5.9)$ \\
\hline Offspring born with malformations & $6250(3.8)$ & $163(4.1)$ & $89(4.4)$ \\
\hline \multicolumn{4}{|l|}{ Maternal psychotropic treatment overlapping pregnancy } \\
\hline 1 dispensation & $2062(1.3)$ & $127(3.2)$ & $51(2.5)$ \\
\hline$\geq 2$ dispensations & $6077(3.7)$ & $388(9.74)$ & $183(9.0)$ \\
\hline \multicolumn{4}{|l|}{ Maternal antidepressant treatment overlapping pregnancy } \\
\hline 1 dispensation & $1852(1.1)$ & $118(3.0)$ & $46(2.3)$ \\
\hline$\geq 2$ dispensations & $3747(2.3)$ & $298(7.5)$ & $134(6.6)$ \\
\hline \multicolumn{4}{|l|}{ Birth year } \\
\hline 2006 & $64771(39.4)$ & $1398(35.1)$ & $873(42.9)$ \\
\hline 2007 & $99721(60.6)$ & $2585(64.9)$ & $1160(57.1)$ \\
\hline \multicolumn{4}{|l|}{ Paternal age (years) at delivery } \\
\hline$<20$ & $957(0.6)$ & $14(0.4)$ & $11(0.5)$ \\
\hline $20-29$ & $46213(28.1)$ & $884(22.2)$ & $496(24.4)$ \\
\hline $30-39$ & $95562(58.1)$ & $2230(56.0)$ & $1094(53.8)$ \\
\hline$\geq 40$ & $21760(13.2)$ & $855(21.5)$ & $432(21.3)$ \\
\hline \multicolumn{4}{|l|}{ Maternal age (years) at delivery } \\
\hline$<20$ & $3205(2.0)$ & $79(2.0)$ & $50(2.5)$ \\
\hline $20-29$ & $69553(42.3)$ & $1518(38.1)$ & $857(42.2)$ \\
\hline $30-39$ & $86363(52.5)$ & $2178(54.7)$ & $1029(50.6)$ \\
\hline$\geq 40$ & $5371(3.3)$ & $208(5.2)$ & $97(4.8)$ \\
\hline Paternal education duration $>9$ years§ & $141667(86.1)$ & $3250(81.6)$ & $1593(78.4)$ \\
\hline Maternal education duration $>9$ years§ & $143579(87.3)$ & $3339(83.8)$ & $1619(79.6)$ \\
\hline \multicolumn{4}{|l|}{ Offspring sex } \\
\hline Male & $84680(51.5)$ & $2039(51.2)$ & $1037(51.0)$ \\
\hline Female & $79812(48.5)$ & $1944(48.8)$ & $996(49.0)$ \\
\hline Gestational age (days; mean (standard deviation)) & $277.6(13.9)$ & $277.2(14.4)$ & $276.8(14.9)$ \\
\hline $\begin{array}{l}\text { Offspring age at end of follow-up (years; mean (standard } \\
\text { deviation)) }\end{array}$ & $7.8(0.6)$ & $7.8(0.6)$ & $7.9(0.6)$ \\
\hline $\begin{array}{l}\text { *Children born to fathers who had no antidepressant dispensati } \\
\text { pregnancy period (B in fig } 1 \text { ). } \\
\text { tChildren born to fathers who received antidepressant treatmen } \\
\text { fChildren born to fathers who initiated antidepressant treatmen } \\
\text { §Details of parental education within seven levels are provided }\end{array}$ & treatment pe & $\begin{array}{l}\text { ping either the c } \\
\text { ig 1). } \\
\text { in fig 1). }\end{array}$ & eriod (A in fig 1) \\
\hline
\end{tabular}

- Exposure to four of the most common SSRI antidepressants (eFigure 8)

- Exclusion of offspring exposed to non-antidepressant psychotropic drugs (eFigure 9)

- Using bootstrap techniques (eFigure 10)

- Exclusion of offspring of mothers receiving psychotropic treatment during pregnancy (eFigure 11)

- Male versus female offspring (eFigure 12)

- Offspring born to fathers receiving continuous antidepressant treatment overlapping conception (eFigure 14)

- Offspring born to fathers receiving no antidepressant treatment from seven to three months before conception (eFigure 15).

Inspection of the Schonfeld residuals did not suggest any violation of the proportional hazards assumption (eFigure 13).

\section{Discussion}

\section{Principal findings}

In this Swedish population based study of 170508 children born in 2006-07 and followed up to the end of 2014, we investigated the effects of paternal antidepressant treatment during conception on offspring preterm birth, malformation, autism, and intellectual disability. We found that children born to fathers receiving antidepressant treatment during conception did not seem to be at a higher risk of these adverse outcomes, although there was evidence of confounding. This finding was consistent in all sensitivity analyses conducted, and was further supported by comparison with the group of children born to fathers who did not receive antidepressant treatment during conception but who initiated treatment during pregnancy. This control comparison group included children who were unlikely to have been conceived from sperm exposed to antidepressant treatment but who were born to fathers who might have shared similar underlying characteristics with fathers receiving antidepressant treatment during conception. We saw a statistically significant reduction in risk of intellectual disability in children born to fathers receiving antidepressant treatment during conception, compared with those born to fathers who initiated treatment later during pregnancy. 


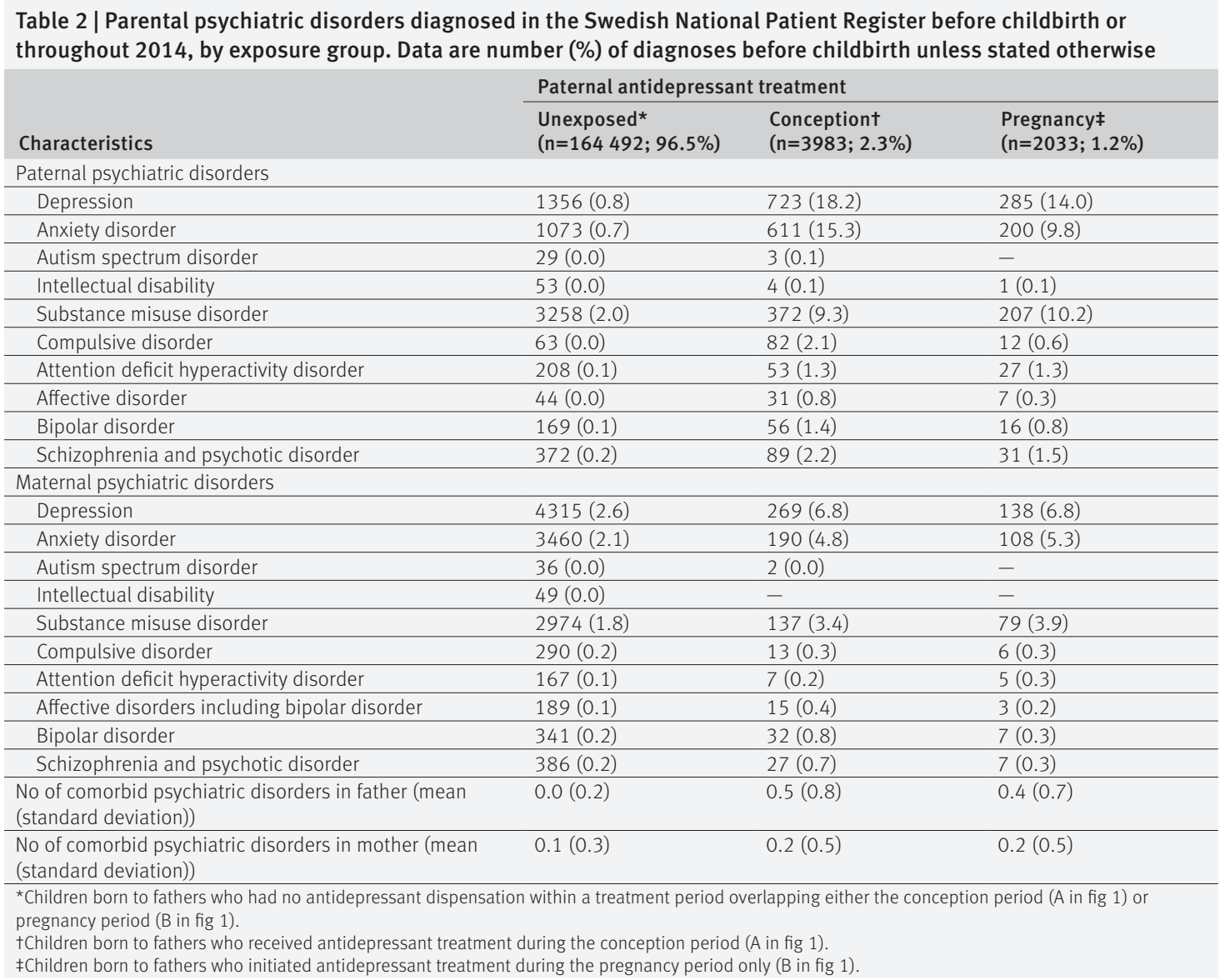

\section{Strengths and limitations}

As a strength, the study included a large, prospective population based cohort of children born during a specific time period, with recent and close to complete coverage of data ( $0.8 \%$ missing data), from a healthcare system with equal access irrespective of ethnicity or geographical location. The independent collection of exposure and outcome data reduced ascertainment bias, and identification of parental diagnoses of psychiatric disorders within several categories allowed a detailed adjustment of psychiatric comorbidity, which is very common. This setting should allow generalisability of the findings beyond the specific population study, and limit the risk of bias by selection or through temporal trends.

However, we cannot rule potential variation of the generalisability of our results in populations that differ from the Swedish population-for example, with respect to variation in cultural diversity and genetic makeup. The Swedish Prescribed Drug Register began on 1 July 2005; if a father had a dispensation of an antidepressant drug just before this date, it would either be undetected if only one dispensation was made, or be defined as treatment that began during pregnancy if there was a follow-up dispensation.

Sensitivity analyses revealed that some children could have been misclassified, but the effect of the misclassification did not alter the finding that paternal antidepressant treatment during conception was not statistically significantly associated with any of the adverse outcomes (eFigures 3-5). Furthermore, adherence to dispensed treatment was not known. However, among fathers who received antidepressant treatment during the conception period, 3450 (86.6\%) had an additional dispensation within six months, suggesting that most were on continuous treatment. Analyses limited to these 3450 fathers revealed results that did not deviate significantly from the main results (eFigure 15).

Additionally, malformation data were obtained from the Swedish Medical Birth Register as a binary variable that includes all malformations according to ICD-10 diagnosis codes of Q00-99, so we were not able to analyse specific malformations. Moreover, our study was limited to livebirths, and thus, any potential effects of paternal antidepressants on fetal survival could not be studied. Although the Swedish Medical Birth Register have stillbirth data, they are limited to stillbirths that occur during the planned delivery. Spontaneous abortions, planned abortions, and stillbirths outside the planned delivery are not captured, which makes the information difficult to interpret.

We also did not have access to psychiatric diagnoses from primary care, which could have contributed to the relatively low number of psychiatric diagnoses 


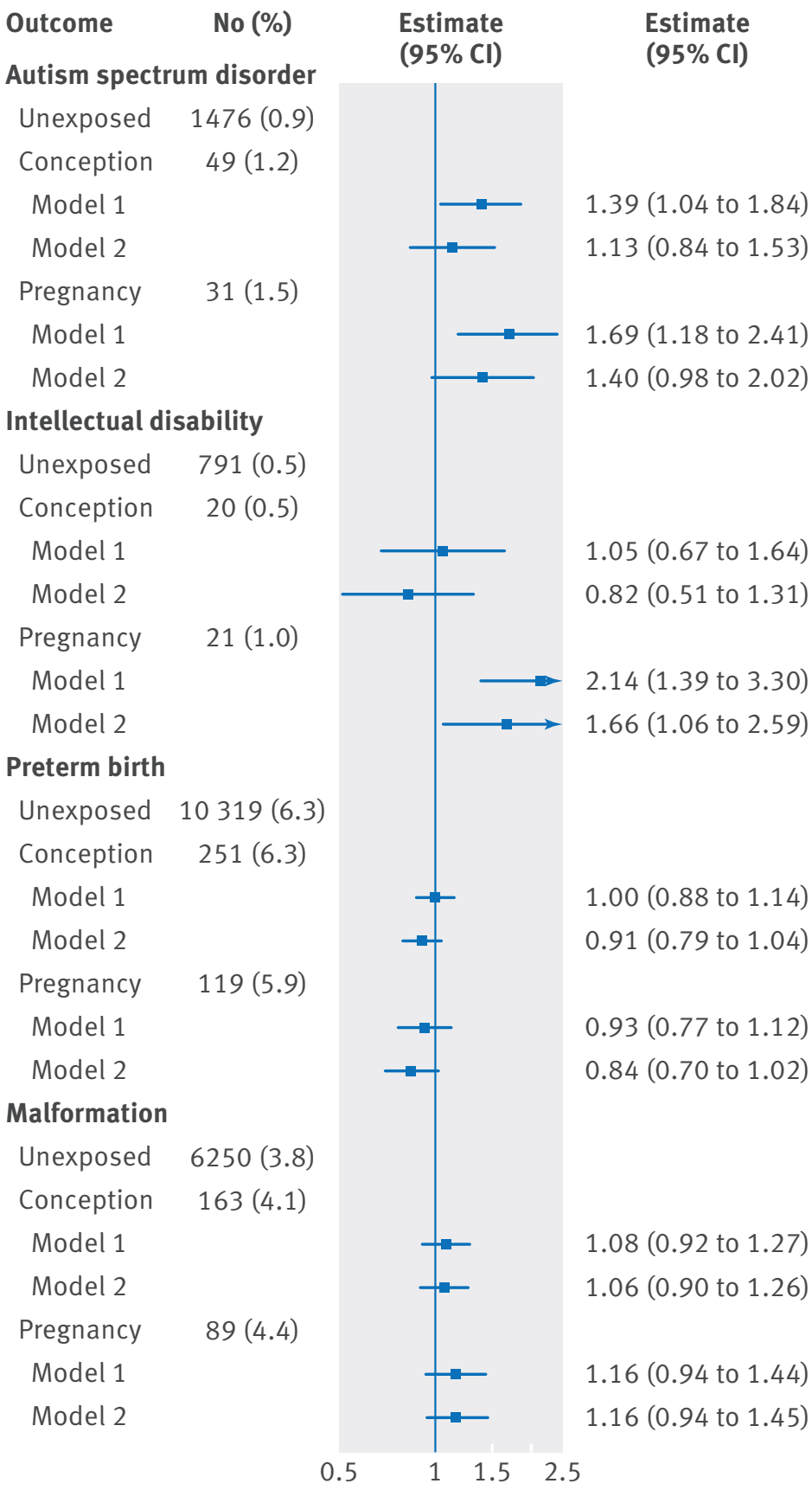

Fig 2 | Risk of adverse outcomes in offspring born to fathers receiving antidepressant treatment during conception or pregnancy periods, versus offspring born to unexposed fathers. Study sample consists of 170508 children conceived from 29 July 2005 and born in 2006-07, and includes three groups: 3983 children born to fathers receiving antidepressant treatment during the conception period (A in fig 1; conception); 2033 children born to fathers initiating antidepressant treatment during the pregnancy period only ( $B$ in fig 1; pregnancy); and 164492 children born to fathers receiving no antidepressant treatment during the conception period ( $A$ in fig 1 ) or pregnancy period ( $B$ in fig 1; unexposed). Figure presents hazard ratios for autism and intellectual disability, odds ratios for preterm birth and malformation, and two sided $95 \%$ confidence intervals in the conception and pregnancy exposure groups versus unexposed group. Model $1=$ unadjusted analyses; model $2=$ analyses adjusted for birthdate; parental age; maternal treatment during pregnancy with psychotropic drugs; parental education level (eTable 4); and diagnosis given to either parent before childbirth (yes/no) within specific psychiatric disorder subgroups including depression, anxiety disorders, substance use disorder, bipolar disorder, compulsive disorder, attention deficit hyperactive disorder, autism spectrum disorder, intellectual disability, and schizophrenia (see eTable 3 for specific diagnosis codes) observed in fathers prescribed antidepressant treatment. Lack of primary care diagnoses is, however, less likely to affect detection of children with autism or intellectual disability, because they are usually referred to a specialist and identified in the Swedish National Patient Register. Lastly, we cannot rule out residual confounding by covariates not included in the analysis-for example, genetics, psychiatric disorder severity, smoking, alcohol consumption, paternal use of non-antidepressant drugs including over-thecounter drugs, maternal folic acid use, emigration, or parental somatic diseases. Unanalysed confounders could be observed from the point estimates that increase and decrease the risk in the analysis. But despite potential residual confounding, the confidence intervals overlapped across all analyses (fig 2).

\section{Comparison with other studies}

In the present study, autism was diagnosed in $0.9 \%$ of children and intellectual disability in $0.5 \%$ of children during the follow-up period of the first seven to eight years after birth, which is similar to the prevalence of $0.6 \%$ for both autism and intellectual disability in children aged 3-10 years in a recent American survey ${ }^{49}$ and prevalence estimates from register based sources in other Scandinavian cohorts. ${ }^{50}$ Similar to a previous study by Yang and colleagues, ${ }^{21}$ we observed an association between paternal antidepressant treatment during conception and autism when potential confounders were not adjusted for, and an attenuation of the association when confounders were adjusted for. But contrary to the Yang study, we did not observe any remaining risk after adjusting for confounders, which might relate to our inclusion of more detailed information about the history of parental psychiatric disorders. This interpretation was further supported by our additional comparison with a negative control group consisting of children unlikely to be exposed to antidepressants during conception but born to fathers who carried similar characteristics associated with the need for antidepressant treatment.

Moreover, in the current study, preterm birth was observed in $6.3 \%$ of children, while malformations according to ICD-10 codes Q00-Q99 were seen in 3.8\% of children. These figures are comparable to those of a study by Engeland and colleagues, who used data from the Medical Birth Registry of Norway; the Norwegian register uses data collection methods similar to those used by the Swedish Medical Birth Register in our study. ${ }^{20}$ Similar to the Engeland study, we observed no association between paternal antidepressant use during conception and offspring malformations. However, although Engeland and colleagues observed a modest increase in risk of preterm birth (adjusted odds ratio 1.3 (95\% confidence interval 1.1 to 1.5$)$ ) in association with fathers receiving SSRI antidepressant treatment during conception, we did not observe an increased risk of preterm birth in association with paternal antidepressant treatment overall (fig 2) or with SSRI antidepressant treatment specifically (eFigure 7). 


\section{Outcome No (\%)}

Autism spectrum disorder

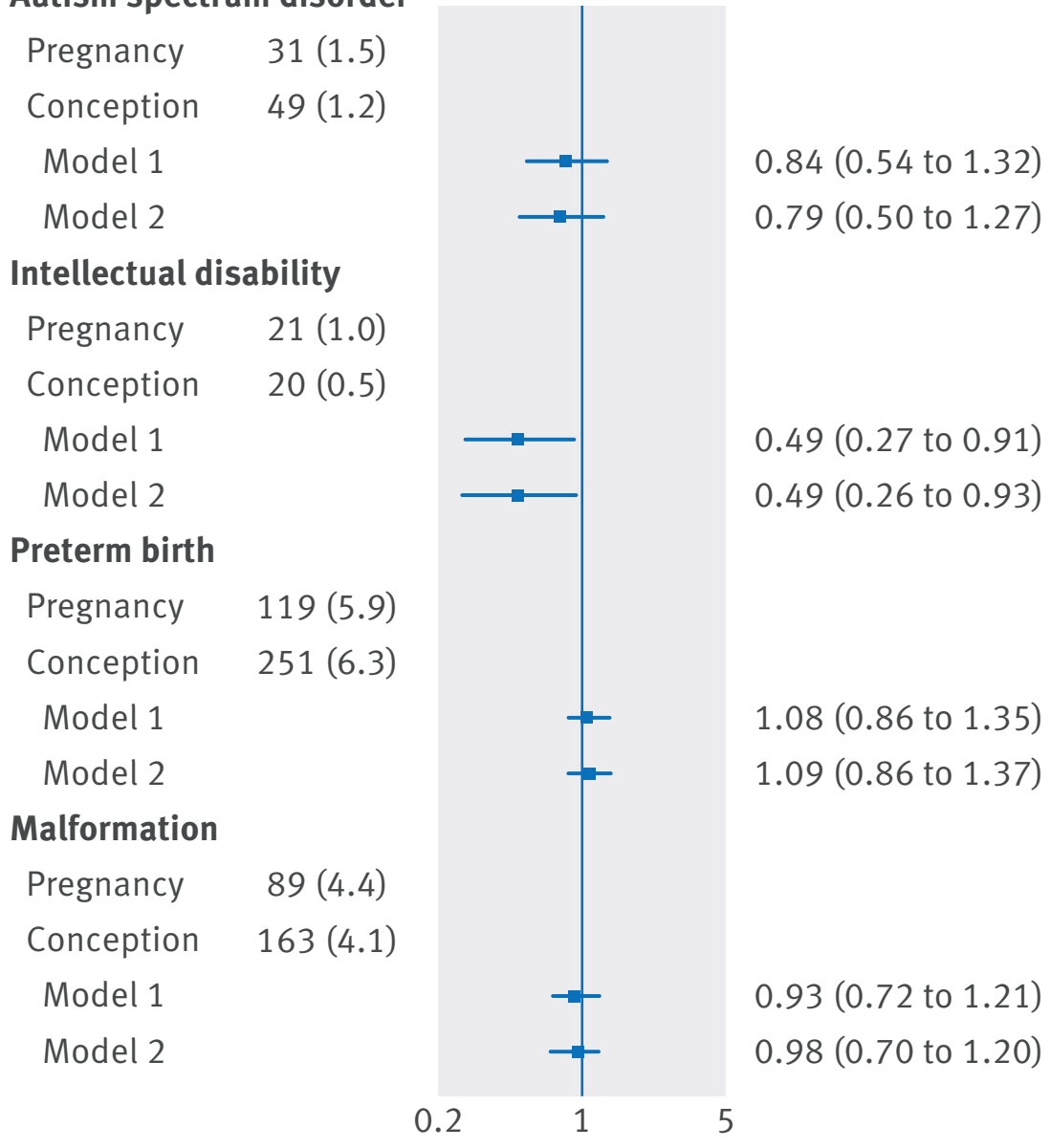

Fig 3 | Risk of adverse outcomes in offspring resulting from paternal antidepressant treatment during conception period versus treatment initiated during pregnancy period. Study sample consists of 6016 children conceived from 29 July 2005 and born in 2006-07, and compares 3983 children born to fathers receiving antidepressant treatment during the conception period (A in fig 1; conception) with 2033 children born to fathers initiating antidepressant treatment during the pregnancy period only ( $B$ in fig 1; pregnancy). Figure presents hazard ratios for autism and intellectual disability, odds ratios for preterm birth and malformation, and two sided $95 \%$ confidence intervals. Model $1=$ unadjusted analyses; model $2=$ analyses adjusted for birthdate; parental age; maternal treatment during pregnancy with psychotropic drugs; parental education level (eTable 4); and diagnosis given to either parent before childbirth (yes/no) within specific psychiatric disorder subgroups including depression, anxiety disorders, substance use disorder, bipolar disorder, compulsive disorder, attention deficit hyperactive disorder, autism spectrum disorder, intellectual disability, and schizophrenia (see eTable 3 for specific diagnosis codes)

\section{Analysis of findings from the present study}

The consistency of our results in the main analysis and sensitivity analyses suggest that paternal antidepressant treatment during conception is not causally related to increased risks of preterm birth, malformations, autism, or intellectual disability in offspring. Instead, our results suggest other factors underlying and confounding the associations between fathers who are treated with antidepressants at conception and the risks of autism and intellectual disability in their offspring. This interpretation is supported by the elevated risks in our crude unadjusted analyses (model 1 in fig 2), which was attenuated by the adjustment for potentially confounding factors.

This interpretation is further supported by results from the analyses of children in the negative control group, who were born to fathers who initiated antidepressant treatment during pregnancy (that is, unlikely to have been exposed to antidepressants at conception but exposed to paternal characteristics similar to those born to fathers receiving antidepressant treatment during conception). The analyses in the negative control group showed similar and even stronger associations in the crude analyses, and a small but statistically significant increased risk of intellectual disability even after adjustment for confounding factors. The elevated risk of intellectual disability was also seen in direct comparison between children born to fathers receiving antidepressant treatment during conception and the negative control group, which further support the importance of the underlying characteristics and not the treatment for the association. These underlying characteristics could be related to dissimilarities between the two exposure groups, for example, relating to the disorder stability.

Antidepressants are used over longer periods of time, and when defining the two mutually exclusive exposure groups, we prioritised the conception period. Therefore, this conception window would include fathers who might just have started their antidepressant treatment, as well as the large number of fathers receiving stable continuous treatment at any given time. By contrast, owing to the mutually exclusive design, fathers who began treatment during pregnancy could not have used antidepressants during conception, and will therefore represent fathers who had experienced an onset or worsening of the underlying disorder that needed the treatment initiation. The observed association between paternal antidepressant treatment beginning during pregnancy and offspring intellectual disability could indicate that having a psychiatric disorder-either through the symptoms or through factors underlying the disorder at this stage of life (eg, genetic susceptibility or life situation)-could constitute a risk factor for offspring autism and intellectual disability.

Furthermore, although our results suggest that antidepressants are not causally associated with the studied outcomes, paternal antidepressant treatment could be a causal factor for other outcomes. And in situations where paternal antidepressant treatment is not thought to be a causal factor, information about paternal treatment could still be an important marker for the underlying factors related to raised risks of autism and intellectual disability in offspring, as shown in our results. Consequently, paternal treatment with antidepressants around the time of conception and pregnancy might be a useful variable in future studies-for example, to capture the onset or worsening of underlying psychiatric illness. Furthermore, our results do not contradict the use of paternal antidepressant treatment as a negative control group when studying the effects of maternal 
antidepressant treatment, given that the outcomes studied are preterm birth, malformations of any kind, autism, and intellectual disability.

\section{Clinical implications}

Depressive illness is a growing health problem worldwide, and antidepressant treatment is increasingly prescribed. At the same time, attention to paternal factors has increased in relation to fetal and offspring health, but research on potential adverse effects on offspring due to paternal antidepressant treatment is limited. Our results suggest that antidepressant use during conception does not increase the risks of preterm birth, malformations, autism, or intellectual disability in offspring. Instead, we observed an increased risk of intellectual disability in children born to fathers who were not treated with antidepressants at the time of conception, but who required antidepressant treatment later during pregnancy.

These results could provide important information to doctors and patients regarding the risks of harming offspring through antidepressant treatment during conception. The information could help families planning to have children and who are concerned about potential risks of paternal treatment on sperm. The results might also be useful to fathers who received antidepressant treatment during the conception of a child who had a preterm birth, malformation, autism, or intellectual disability, and who might be blaming themselves unnecessarily. However, research on this topic is limited, and although the present study did not find a causal link between the studied outcomes and antidepressant treatment, replication of the results is imperative. Furthermore, the results reflect effects on a group level, and might not be generalisable to all individuals and populations.

Contributors: SS initiated the collaborative project. AV, AR, and SS collected, assembled, and quality controlled the data. SS and AR are the guarantors. AV, MA, SZL, and SS conceived and designed the study and wrote the statistical analysis plan before the start of data analysis. AV developed the statistical analysis programs and analysed the data. $A V$ and SS drafted the first version of the report. All authors analysed and interpreted the data. All authors contributed to the content and critical revision of the report and agreed to submit the report for publication. The corresponding author attests that all listed authors meet authorship criteria and that no others meeting the criteria have been omitted.

Funding: This study was supported by grants from the National Institutes of Health; grant HD073978 from the Eunice Kennedy Shriver National Institute of Child Health and Human Development, National Institute of Environmental Health Sciences, and National Institute of Neurological Disorders and Stroke; grant MH097849 from the National Institute of Mental Health; the Beatrice and Samuel A Seaver Foundation (SS is a Seaver Faculty Scholar); the Fredrik and Ingrid Thuring Foundation (AV); and the Swedish Society of Medicine (AV). No funder had any role in the study design; data collection, analysis, or interpretation; in the writing of the report; or in the decision to submit the article for publication. The views expressed are those of the authors and not necessarily those of the funders, or the organisations they represent.

Competing interests: All authors have completed the ICMJE uniform disclosure form at www.icmje.org/coi_disclosure.pdf and declare: support from the National Institutes of Health, Beatrice and Samue A Seaver Foundation, Fredrik and Ingrid Thuring Foundation, and Swedish Society of Medicine for the submitted work; no financial relationships with any organisations that might have an interest in the submitted work in the previous three years; no other relationships or activities that could appear to have influenced the submitted work.

Ethical approval: The study was approved by the regional ethics committee in Stockholm, Sweden (2012/1548/31-1). No individual level consent was required, and all data used were anonymised.

Data sharing: No additional data available.

The lead author affirms that the manuscript is an honest, accurate, and transparent account of the study being reported; that no important aspects of the study have been omitted; and that any discrepancies from the study as planned (and, if relevant, registered) have been explained.

This is an Open Access article distributed in accordance with the Creative Commons Attribution Non Commercial (CC BY-NC 4.0) license, which permits others to distribute, remix, adapt, build upon this work non-commercially, and license their derivative works on different terms, provided the original work is properly cited and the use is noncommercial. See: http://creativecommons.org/licenses/by-nc/4.0/.

1 Sandin S, Hultman CM, Kolevzon A, Gross R, MacCabe JH, Reichenberg A. Advancing maternal age is associated with increasing risk for autism: a review and meta-analysis. J Am Acad Child Adolesc Psychiatry 2012;51:477-486.e1. doi:10.1016/j. jaac.2012.02.018

2 McGrath JJ, Petersen L, Agerbo E, Mors O, Mortensen PB, Pedersen CB. A comprehensive assessment of parental age and psychiatric disorders. JAMA Psychiatry 2014;71:301-9. doi:10.1001/ jamapsychiatry.2013.4081

3 Levine SZ, Kodesh A, Viktorin A, et al. Association of maternal use of folic acid and multivitamin supplements in the periods before and during pregnancy with the risk of autism spectrum disorder in offspring. JAMA Psychiatry 2018;75:176-84. doi:10.1001/ jamapsychiatry.2017.4050

4 Viktorin A, Uher R, Reichenberg A, Levine SZ, Sandin S. Autism risk following antidepressant medication during pregnancy. Psychol Med 2017:47:2787-96. doi:10.1017/S0033291717001301

5 Viktorin A, Uher R, Kolevzon A, Reichenberg A, Levine SZ, Sandin S. Association of antidepressant medication use during pregnancy with intellectual disability in offspring. JAMA Psychiatry 2017;74:1031-8. doi:10.1001/ jamapsychiatry.2017.1727

6 Rai D, Lee BK, Dalman C, Golding J, Lewis G, Magnusson C. Parental depression, maternal antidepressant use during pregnancy, and risk of autism spectrum disorders: population based case-control study. BMJ 2013:346:f2059. doi:10.1136/bmi.f2059

7 Sujan AC, Rickert ME, Öberg AS, et al. Associations of maternal antidepressant use during the first trimester of pregnancy with preterm birth, small for gestational age, autism spectrum disorder, and attention-deficit/hyperactivity disorder in offspring. JAMA 2017;317:1553-62. doi:10.1001/jama.2017.3413

8 Hviid A, Melbye M, Pasternak B. Use of selective serotonin reuptake inhibitors during pregnancy and risk of autism. N Engl J Med 2013;369:2406-15. doi:10.1056/NEJMoa1301449

9 Liu X, Agerbo E, Ingstrup KG, et al. Antidepressant use during pregnancy and psychiatric disorders in offspring: Danish nationwide register based cohort study. BMJ 2017;358:j3668. doi:10.1136/ bmj.j3668

10 Viktorin A, Lichtenstein P, Lundholm C, et al. Selective serotonin re-uptake inhibitor use during pregnancy: association with offspring birth size and gestational age. Int J Epidemiol 2016;45:170-7. doi:10.1093/ije/dyv351

11 Figueroa R. Use of antidepressants during pregnancy and risk of attention-deficit/hyperactivity disorder in the offspring. I Dev Behav Pediatr 2010:31:641-8 doi:10.1097/DBP0b013e3181e5ac93

12 Rai D, Lee BK, Dalman C, Newschaffer C, Lewis G, Magnusson C. Antidepressants during pregnancy and autism in offspring: population based cohort study. BMJ 2017;358:j2811. doi:10.1136/ bmj.j2811

13 Sørensen MJ, Grønborg TK, Christensen J, et al. Antidepressant exposure in pregnancy and risk of autism spectrum disorders. Clin Epidemiol 2013;5:449-59. doi:10.2147/CLEP.S53009

14 Palmsten K, Hernández-Díaz S, Huybrechts KF, et al. Use of antidepressants near delivery and risk of postpartum hemorrhage: cohort study of low income women in the United States. BMJ 2013;347:f4877. doi:10.1136/bmj.f4877

15 Palmsten K, Setoguchi S, Margulis AV, Patrick AR, Hernández-Díaz S. Elevated risk of preeclampsia in pregnant women with depression: depression or antidepressants? Am J Epidemiol 2012;175:988-97. doi:10.1093/aje/kwr394

16 Pedersen LH, Henriksen TB, Vestergaard M, Olsen J, Bech $\mathrm{BH}$. Selective serotonin reuptake inhibitors in pregnancy and congenital malformations: population based cohort study. BMJ 2009;339:b3569. doi:10.1136/bmj.b3569 
17 World Health Organization. Depression and other common mental disorders: global health estimates. 2017. www.who.int/ mental_health/management/depression/prevalence_global_health_ estimates/en/

18 Abbing-Karahagopian V, Huerta C, Souverein PC, et al. Antidepressant prescribing in five European countries: application of common definitions to assess the prevalence, clinical observations, and methodological implications. Eur J Clin Pharmacol 2014;70:84957. doi:10.1007/s00228-014-1676-z

19 Abbasi J. The paternal epigenome makes its mark JAMA 2017;317:2049-51. doi:10.1001/jama.2017.1566

20 Engeland A, Bjørge T, Daltveit AK, et al. Effects of preconceptional paternal drug exposure on birth outcomes: cohort study of 340000 pregnancies using Norwegian population-based databases. BrJ Clin Pharmacol 2013;75:1134-41. doi:10.1111/j.13652125.2012.04426.x

21 Yang F, Chen J, Miao MH, et al. Risk of autism spectrum disorder in offspring following paternal use of selective serotonin reuptake inhibitors before conception: a population-based cohort study. BM/ Open 2017;7:e016368. doi:10.1136/bmjopen-2017-016368

22 Herati AS, Zhelyazkova BH, Butler PR, Lamb DJ. Age-related alterations in the genetics and genomics of the male germ line. Fertil Steril 2017;107:319-23. doi:10.1016/j.fertnstert.2016.12.021

23 Kumar VS, Sharma VL, Tiwari P, et al. The spermicidal and antitrichomonas activities of SSRI antidepressants. Bioorg Med Chem Lett 2006:16:2509-12 doi:10.1016/j.bmcl.2006.01.078

24 Olfson M, Marcus SC. National patterns in antidepressant medication treatment. Arch Gen Psychiatry 2009;66:848-56. doi:10.1001/archgenpsychiatry.2009.81

25 Koyuncu H, Serefoglu EC, Yencilek E, Atalay H, Akbas NB, Sarıca K. Escitalopram treatment for premature ejaculation has a negative effect on semen parameters. Int J Impot Res 2011;23:257-61. doi:10.1038/iiir.2011.35

26 Tanrikut C, Feldman AS, Altemus M, Paduch DA, Schlegel PN Adverse effect of paroxetine on sperm. Fertil Steril 2010;94:1021-6. doi:10.1016/i.fertnstert.2009.04.039

27 Tanrikut C, Schlegel PN. Antidepressant-associated changes in semen parameters. Urology 2007;69:185.e5-7. doi:10.1016/ j.urology.2006.10.034

28 Elnazer HY, Baldwin DS. Treatment with citalopram, but not with agomelatine, adversely affects sperm parameters: a case report and translational review. Acta Neuropsychiatr 2014;26:125-9. doi:10.1017/neu.2013.60

29 Borini A, Tarozzi N, Bizzaro D, et al. Sperm DNA fragmentation: paternal effect on early post-implantation embryo development in ART. Hum Reprod 2006;21:2876-81. doi:10.1093/humrep/del251

30 Olsen AK, Andreassen A, Singh R, et al. Environmental exposure of the mouse germ line: DNA adducts in spermatozoa and formation of de novo mutations during spermatogenesis. PLOS One 2010;5:e11349. doi:10.1371/journal.pone.0011349

31 Iossifov I, O'Roak BJ, Sanders SJ, et al. The contribution of de novo coding mutations to autism spectrum disorder. Nature 2014:515:216-21. doi:10.1038/nature13908

32 Lim ET, Uddin M, De Rubeis S, et al, Autism Sequencing Consortium. Rates, distribution and implications of postzygotic mosaic mutations in autism spectrum disorder. Nat Neurosci 2017;20:1217-24. doi: $10.1038 / n n .4598$

33 Hultman CM, Sandin S, Levine SZ, Lichtenstein P, Reichenberg A. Advancing paternal age and risk of autism: new evidence from a population-based study and a meta-analysis of epidemiological studies. Mol Psychiatry 2011;16:1203-12. doi:10.1038/ mp.2010.121
34 Cnattingius S, Ericson A, Gunnarskog J, Källén B. A quality study of a medical birth registry. Scand J Soc Med 1990;18:143-8. doi:10.1177/140349489001800209

35 Høgberg U, Larsson N. Early dating by ultrasound and perinatal outcome. A cohort study. Acta Obstet Gynecol Scand 1997;76:90712. doi:10.3109/00016349709034900

36 Ekbom A. The Swedish Multi-generation Register. Methods Mol Biol 2011;675:215-20, doi:10.1007/978-1-59745-423-0 10

37 Statistics Sweden. Multi-generation register 2016 - A description of contents and quality. Statistics Sweden, 2017

38 World Health Organization. The anatomical therapeutic chemical classification system with defined daily doses (ATC/DDD). WHO, 2006.

39 Wettermark B, Hammar N, Fored CM, et al. The new Swedish Prescribed Drug Register--opportunities for pharmacoepidemiological research and experience from the first six months [correction in: Pharmacoepidemiol Drug Saf 2008;17:533]. Pharmacoepidemiol Drug Saf 2007:16:726-35. doi:10.1002/pds.1294

40 Baarends WM, van der Laan R, Grootegoed JA. DNA repair mechanisms and gametogenesis. Reproduction 2001;121:31-9. doi:10. /rep.0.1210031

41 Ludvigsson JF, Andersson E, Ekbom A, et al. External review and validation of the Swedish national inpatient register. BMC Public Health 2011:11:450, doi:10.1186/1471-2458-11-450

42 Sandin S, Schendel D, Magnusson P, et al. Autism risk associated with parental age and with increasing difference in age between the parents. Mol Psychiatry 2016:21:693-700. doi:10.1038 mp. 2015.70

43 Atladottir HO, Gyllenberg D, Langridge A, et al. The increasing prevalence of reported diagnoses of childhood psychiatric disorders: a descriptive multinational comparison. Eur Child Adolesc Psychiatry 2015;24:173-83. doi:10.1007/s00787-014-0553-8

44 Lee SH, Ripke S, Neale BM, et al, Cross-Disorder Group of the Psychiatric Genomics ConsortiumInternational Inflammatory Bowe Disease Genetics Consortium (IIBDGC). Genetic relationship between five psychiatric disorders estimated from genome-wide SNPS. Nat Genet 2013;45:984-94. doi:10.1038/ng.2711

45 Bulik-Sullivan B, Finucane HK, Anttila V, et al, ReproGen ConsortiumPsychiatric Genomics ConsortiumGenetic Consortium for Anorexia Nervosa of the Wellcome Trust Case Control Consortium 3. An atlas of genetic correlations across human diseases and traits. Nat Genet 2015;47:1236-41. doi:10.1038/ng.3406

46 Korn EL, Graubard BI, Midthune D. Time-to-event analysis of longitudinal follow-up of a survey: choice of the time-scale. Am J Epidemiol 1997;145:72-80. doi:10.1093/oxfordjournals.aje. a009034

47 Efron B, Tibshirani RJ. An introduction to the bootstrap. CRC press, 1994.

48 Grambsch PM, Therneau TM. Proportional hazards tests and diagnostics based on weighted residuals. Biometrika 1994;81:51526. doi:10.1093/biomet/81.3.515.

49 Boyle CA, Boulet S, Schieve LA, et al. Trends in the prevalence of developmental disabilities in US children, 1997-2008. Pediatrics 2011;127:1034-42. doi:10.1542/peds.2010-2989

50 Maulik PK Mascarenhas MN Mathers CD Dua T, Saxena S. Prevalence of intellectual disability: a meta-analysis of populationbased studies. Res Dev Disabil 2011;32:419-36. doi:10.1016/j. ridd.2010.12.018

Web appendix: Supplementary material 